\title{
Situation Actuelle Et Facteurs Influençant L'allaitement Dans La Ville De Rabat Au Maroc A Propos D'une Enquête Chez 275 Mères
}

\author{
Zineb Sqalli Houssaini \\ Sultana Inekac \\ Laboratoire de Biochimie et Neurosciences, Faculté des Sciences et \\ Techniques Settat BP 57726000, Settat, Maroc \\ Maryam Benbachir Hassani \\ Laboratoire de Biochimie et Neurosciences, Faculté des Sciences et \\ Techniques Settat BP 57726000, Settat, Maroc \\ Mohammed Ouhssine \\ Zineb Guessous \\ Laboratoire de Biotechnologie, Environnement et Qualité, Faculté \\ des Sciences BP 133 14000, Kenitra, Maroc
}

\begin{abstract}
Breastfeeding is the most ideal type of feeding for all infants; it is the optimal food for both newborns and young infants. It has no equal as it is adjusted to their needs and their abilities. Breastfeeding has many advantages for the child as well as the mother. The main objective of this study is to evaluate the current situation of breastfeeding in the region of Rabat city; assess the prevalence of breastfeeding and the different methods adopted; and identify the rationale behind the choice of each feeding form, the weaning methods, and the age of diversification of the child's diet.

To meet these objectives, a survey was conducted through a questionnaire of 31 questions on a sample of 275 women with children aged between 0 and 3 years and thus with a recent breastfeeding experience.

- The women surveyed were aged between 18 and 38 years, $55 \%$ are under 30 years.

- $95 \%$ of women surveyed are from a medium or low social economical class background, $42.5 \%$ of them are illiterate, $16 \%$ of them have a primary or secondary school level, while $41.5 \%$ have a higher level of education.

- Primiparous represent $32 \%$ of participants, while $68 \%$ are multiparous. The average number of children per woman is 2.21 .
\end{abstract}


- The prevalence of breastfeeding represents $89 \% .40 \%$ of participants have opted for exclusive breastfeeding while almost $50 \%$ have chosen mixed feeding. Finally, $11 \%$ of participants have adopted artificial feeding.

- Only $34 \%$ of respondents could breastfeed their newborn during the first 24 hours after delivery, and more than half of the respondents $(54 \%)$ did it only the second day post-delivery.

- $\quad$ The average duration of breastfeeding is 7.7 months

- Many factors that could impact the choice of the breastfeeding mode were studied during this survey. The inter-factorial analysis showed that the delivery mode, the socio-professional class, the level of education, and the sex of children can have a significant influence on the mode and the frequency of breastfeeding.

- $80 \%$ of women have already weaned their infants through different ways. The forecast of weaning from breastfeeding diverges according to the breastfeeding mode chosen. The current average age of weaning predicted is estimated to 6.9 months.

- The average age of introducing another feeding alternative which is different from breastfeeding (water, orange juice, formula milk etc.) is estimated to $2.38 \%$ months. It diverges depending on the type of food that was introduced.

- The average age of introducing industrial milk is estimated to 3 months accompanied by 3 feeding bottles per day on an average.

To face the exclusive breastfeeding regression tendency and the very early introduction of formula milk, a promotional awareness campaign on breastfeeding of mothers is compulsory.

The factors identified to be influencing the mode of breastfeeding represent an alternative way to be examined. This is for the purpose of elaborating direct actions of the promotion of breastfeeding for women with low willingness to breastfeed.

Keywords: Breastfeeding, survey, socio-professional classes, level of education, feeding mode, exclusive breastfeeding, mixed feeding, artificial feeding, weaning

\section{Résumé}

L'allaitement au sein est le type idéal d'alimentation pour tous les nourrissons, il constitue l'aliment optimal du nouveau-né et du jeune nourrisson; il n'a pas d'égal car il est ajusté à ses besoins comme à ses capacités. Il présente de nombreux avantages, tant pour l'enfant que pour la mère (Lara-Villoslada et al., 2007). 
L'objectif principal de cette étude est d'évaluer la situation actuelle de l'allaitement maternel au niveau de la ville de Rabat. Les objectifs secondaires sont d'évaluer la prévalence de l'allaitement maternel et des différents modes d'allaitement adoptés, cerner les motivations influençant le choix de chaque mode d'allaitement, les prévisions ou modalités de sevrage, l'âge de la diversification de l'alimentation de l'enfant.

Pour répondre à ces objectifs, une enquête a été réalisée sur la base d'un questionnaire de 31 questions, auprès de 275 femmes ayant des enfants âgés de 0 à 3 ans et ayant donc une expérience récente d'allaitement.

- Les femmes interrogées sont âgés de 18 à 38 ans dont plus de la moitié ont moins de 30 ans (55\%).

- 95\% des femmes interrogées sont issues d'un milieu à niveau socioprofessionnels moyen (C) ou faible (DE), 42,5\% des femmes questionnées sont analphabètes, $16 \%$ d'entre elles ont un niveau primaire ou secondaire tandis que $41,5 \%$ des femmes ont un niveau d'instruction supérieur.

- Les primipares constituent 32\% des femmes interrogées tandis que $68 \%$ sont multipares. le nombre d'enfants par femme s'élève à 2,21 .

- La prévalence de l'allaitement maternel représente $89 \%$. Ainsi, $40 \%$ des femmes interrogées ont opté pour l'allaitement maternel exclusif tandis que près de la moitié ont opté pour l'allaitement mixte. Enfin, les femmes ayant adopté l'allaitement artificiel représentent $11 \%$ des enquêtées.

- Concernant la première mise au sein, 34\% seulement des femmes interrogées ont pratiqué l'allaitement pendant les premières 24 heures après l'accouchement alors que plus de la moitié des femmes interrogées (54\%) n'ont donnent le sein que le lendemain de l'accouchement.

- La durée moyenne de l'allaitement s'élève à 7,7 mois.

- Plusieurs facteurs susceptibles d'influencer le choix du mode d'allaitement ont été étudiés lors de cette enquête. L'analyse interfactorielle fait ressortir que le mode d'accouchement, la classe socioprofessionnelle, le niveau d'instruction et le sexe de l'enfant influencent de manière significative le mode et la fréquence de l'allaitement maternel.

- $80 \%$ des femmes ont déjà sevré leurs nourrissons par différentes manières. Les prévisions de sevrage des femmes allaitantes différent selon le mode d'allaitement choisi. Ainsi, l'âge moyen de sevrage effectif et/ou envisagé est estimé à 6,9 mois.

- L'âge moyen d'introduction d'une alimentation autre que le lait maternel (eau, jus d'orange, compote de fruit, lait industriel..) s'élève à 2,38 mois. Il diffère selon le type d'aliment introduit. L'âge moyen d'introduction 
du lait industriel quant à lui est estimé à 3 mois avec en moyenne

3 biberons par jour.

Face à la tendance à la régression de la pratique de l'allaitement maternel exclusif en rapport avec l'introduction de plus en plus précoce du lait industriel, un travail de promotion de l'allaitement maternel et de sensibilisation des mères s'avère indispensable.

Les facteurs identifiés comme influençant le mode d'allaitement peuvent alors représenter des pistes à exploiter pour élaborer des actions ciblées de promotion de l'allaitement au profit des mères les moins enclines à allaiter.

Mots clés: Lait maternel, enquête, classe socioprofessionnelle (CSP), niveau d'instruction, mode d'allaitement, allaitement maternel exclusif, allaitement mixte, allaitement artificiel, sevrage

\section{Introduction}

L'allaitement maternel exclusif est une méthode d'alimentation des nourrissons largement recommandée dans le monde entier. Il fournit au nourrisson tout ce dont il a besoin : chaleur, affection, éléments nutritifs, protection immunitaire (Elbakali, 2011).

En mai 2001, L’Organisation Mondiale de la Santé (OMS) a préconisé que 1'allaitement exclusif était le régime alimentaire optimal des enfants, il signifie d'alimenter le nourrisson uniquement avec du lait maternel et rien d'autre, pas même de l'eau, pendant six mois, puis continuer à l'allaiter et lui donner des aliments complémentaires appropriés jusqu'à l'âge de deux ans et plus (Organisation Mondiale de la Santé, 2001).

Ces dernières décennies, nombre de travaux scientifiques ont conclu à un effet bénéfique de l'allaitement sur la santé de la mère et de l'enfant. Ils soulignent les effets positifs à long terme de l'allaitement, notamment sur la réduction du risque infectieux et le développement cognitif et moteur de l'enfant (Duijts et al., 2009; Kramer et al., 2008). Chez les mères, l'allaitement réduirait l'incidence des cancers du sein et améliorerait la santé en suite de couches (Labbok, 2001).

Dans plusieurs pays industrialisés, la pratique de l'allaitement au sein a repris de la vigueur. A titre d'exemple, la prévalence de l'allaitement exclusif a atteint par exemple 95\% au Danemark, 90\% en Pologne ou encore $89 \%$ en Suède (Kersuzan et al., 2014).

Pourtant, une réelle sonnette d'alarme est tirée dans d'autres pays face au déclin observé concernant la pratique de l'allaitement maternel (Barkat et al., 2004). En effet, on assiste actuellement à une tendance à la régression de cette pratique en rapport avec le progrès dans la fabrication et la commercialisation des laits industriels et avec le manque d'information et de sensibilisation des mères. 
$\mathrm{Au}$ Maroc aussi, la situation se dégrade comme en témoignent les données successives des enquêtes nationales sur la population et la santé de 1992,1997à 2004 où le taux de l'allaitement maternel exclusif à l'âge de 6 mois est passé respectivement de 51\%, 41\% à 32\% (Ministère de la santé au Maroc, 2005).

Dans ce contexte, nous nous proposons de réaliser une enquête auprès d'un groupe de mères d'enfants âgés entre 0 et 3 ans, ayant donc une expérience d'allaitement récente. Les objectifs sont de connaître la prévalence, la durée et les pratiques de l'allaitement maternel ainsi que les facteurs influençant le mode d'allaitement d'une part, et de connaitre les conditions de diversification alimentaire et les modalités de sevrage d'autre part.

\section{Methodes de l'enquete}

Objectif de l'enquête

\section{L'objectif principal}

L'objectif principal de cette enquête est d'évaluer les pratiques des mères à l'égard de l'allaitement maternel et de cerner les modalités de sevrage, au niveau de la ville de Rabat.

\section{Les objectifs spécifiques}

Les objectifs spécifiques assignés à l'enquête sont:

1. Définir la prévalence et la durée de l'allaitement maternel;

2. Identifier les pratiques relatives à la première mise au sein;

3. Identifier les facteurs influençant le choix du mode d'allaitement (Age, CSP, niveau d'instruction, mode d'accouchement, expériences antérieures d'allaitement);

4. Connaître l'expérience d'allaitement des mères et son influence sur les pratiques ultérieures d'allaitement;

5. Déterminer les conditions de diversification alimentaire dispensée aux nourrissons notamment connaitre l'âge, la fréquence et les raisons d'introduction d'une alimentation autre que le lait maternel;

6. Déterminer les modalités de sevrage des nourrissons.

\section{Population cible et lieu d'administration de l'enquête}

La population cible est composée de mères d'enfants âgés de 0 à 3 ans. Les femmes considérées sont au nombre de 275. Ce sont des femmes primipares et multipares dont l'âge varie entre 18 et 38 ans et qui disposent d'une expérience d'allaitement récente.

Lors du recrutement des répondants, l'échantillon a été sélectionné de manière aléatoire. Toutes les femmes interrogées ont été inclues dans l'échantillon sans prendre en compte leur âge, Catégorie Socio- 
professionnelle (CSP) ou parité. Un seul critère d'inclusion a été pris en compte pour la sélection de l'échantillon à savoir disposer d'une expérience d'allaitement dans les trois dernières années.

Toutefois, lors du traitement des données, nous avons procédé à un redressement de l'échantillon en termes de CSP et d'âge, de manière à refléter les proportions réelles de l'univers étudié, en l'occurrence la ville de Rabat. La pondération a été effectuée sur la base des données du recensement de 2004.

Le questionnaire a été administré dans divers lieux à savoir des cabinets médicaux de gynécologie et de pédiatrie, les deux maternités ainsi que dans différents centres de santé de la ville de Rabat et ce, après l'autorisation des personnes et entités concernées.

Parmi les femmes sollicitées, trois d'entre elles ont refusé de participer à l'étude.

\section{Elaboration du Questionnaire}

Pour la réalisation de l'enquête, nous avons conçu un questionnaire contenant 31 questions dont 14 questions ouvertes, 14 questions fermées à choix unique et 3 questions fermées à choix multiples.

Le questionnaire est conçu pour permettre de recueillir les informations sur le profil socio-économique et biodémographique des mères, le mode et le lieu d'accouchement, le mode d'allaitement adopté par les enquêtés, les pratiques de la première tétée, les motivations du choix de chaque mode d'allaitement, les pratiques d'allaitement, les freins à l'allaitement maternel ainsi que l'âge, les prévisions et les modalités de sevrage des nourrissons.

\section{Traitement et analyse des données}

\section{Recueil des données}

Nous avons réalisé cette enquête dans la ville de Rabat durant la période allant du 1erjanvier au 31 juillet 2014.

Le questionnaire a été administré en face à face papier. La durée moyenne d'administration du questionnaire est de 45 minutes.

\section{Analyse statistique}

Les données statistiques ont été traitées à l'aide du logiciel SPSS. Il s'agit d'un logiciel d'enquête et d'analyse des données. Il permet de traiter quantitativement et qualitativement les données. Les résultats sont exprimés en pourcentage ou en valeur absolue. Ce type de logiciel a également permis une analyse croisée des résultats. 


\section{Limites de l'enquête}

Le choix d'une enquête par questionnaire composé de 14questions ouvertes, ne conditionne certes pas les réponses des femmes interrogées et a même permis de générer des réponses plus larges et variées, mais a engendré néanmoins quelques difficultés d'analyse des réponses.

\section{RESULTATS ET DISCUSSIONS}

\section{Répartition de la population selon le critère d'inclusion}

- Il ressort de l'enquête que la prévalence de l'allaitement maternel est de $89 \%$ dont $49 \%$ d'allaitement mixte et $40 \%$ seulement d'allaitement maternel exclusif. L'allaitement artificiel a été adopté par $11 \%$ des femmes interrogées.

\begin{tabular}{|lc|}
\hline Mode d'allaitement & Pourcentage \\
\hline Allaitement artificiel & $11 \%$ \\
\hline Allaitement mixte & $49 \%$ \\
\hline $\begin{array}{l}\text { Allaitement maternel } \\
\text { exclusif }\end{array}$ & $40 \%$ \\
\hline
\end{tabular}

Tableau 1 : répartition par mode d'allaitement

Les résultats de F. Bellati-Saadif et al. (1996), ayant réalisé une enquête en 1996 auprès de 220 mères pour cerner la situation de l'allaitement maternel dans la région d'Agadir fait ressortir quant à elle une prévalence de l'allaitement maternel de $84 \%$ dont $66 \%$ d'allaitement exclusif et $18 \%$ d'allaitement mixte.

- Une enquête prospective réalisée par A. Hassani et al. (2005) auprès de 211 couples mère nouveau-nés ayant séjourné à la maternité Souissi de Rabat a révélé quant à elle une prévalence de l'allaitement maternel de 91\%. En concordance avec les résultats de ces enquêtes, il ressort de notre enquête que la prévalence de l'allaitement maternel qui s'élève à $89 \%$ n'a pas connu une de variations notoires durant ces deux dernières décennies puisque cette variation n'excède pas un écart de \pm 5 points au cours d'une trentaine d'année.

En concordance avec l'enquête de F. Bellati-Saadif et al. (1996), certes la prévalence de l'allaitement maternel a augmenté de 5 points mais on note une forte progression de l'allaitement mixte au détriment de l'allaitement exclusif qui aurait connu une baisse notoire de 26 points passant de $66 \%$ à $40 \%$. Ceci représente qui représente un point de vigilance au vu de ses bienfaits indéniables pour la mère et le nourrisson.

\section{Caractéristiques de la population}

- Plus de la moitié des femmes interrogées sont âgées de moins de 30 ans.

- $42 \%$ des femmes interrogées sont analphabètes. 
- $29 \%$ des femmes interviewées sont mères au foyer. Les autres femmes sont notamment fonctionnaires, employés ou occupent des professions libérales.

\begin{tabular}{|ll|}
\hline Age : & \multicolumn{1}{|c|}{ Pourcentage } \\
\hline$<20$ ans & $6,5 \%$ \\
\hline $20-25$ ans & $21,5 \%$ \\
\hline 26-30ans & $26,5 \%$ \\
\hline 31-35ans & $21,8 \%$ \\
\hline$>35$ ans & $23,6 \%$ \\
\hline Niveau d'études : \\
\hline Analphabète & $42,5 \%$ \\
\hline $\begin{array}{l}\text { Primaire / } \\
\text { secondaire }\end{array}$ & $16,0 \%$ \\
\hline Niveau Supérieur & $41,5 \%$ \\
\hline Niveau Socioéconomique : \\
\hline AB_Elévé & $5,1 \%$ \\
\hline C_Moyen & $52,0 \%$ \\
\hline DE_Faible & $42,9 \%$ \\
\hline
\end{tabular}

Tableau 2 : répartition par critères sociodémographiques

- La majorité des femmes enquêtées appartiennent à la CSP moyenne et faible avec respectivement, $52 \%$ et $43 \%$. A noter que la définition de la classe socioprofessionnelle à laquelle appartient les interviewées a été déterminée en prenant en compte plusieurs variables notamment le niveau d'instruction, la profession et le quartier de résidence.

\section{Caractéristiques_d"'accouchement}

- $68 \%$ des parturientes sont multipares. le nombre d'enfants par femme s'élève à 2,21 .

- $57 \%$ des femmes ont donné naissance à des enfants de sexe masculin et $43 \%$ des femmes interrogées ont accouché d'enfants de sexe féminin.

\begin{tabular}{|ll|}
\hline Parité : & $\%$ \\
\hline Primipare & $32,0 \%$ \\
\hline Multipare & $68,0 \%$ \\
\hline Sexe de l'enfant : & $\%$ \\
\hline Masculin & $32,0 \%$ \\
\hline Féminin & $68,0 \%$ \\
\hline Lieu d'accouchement : & $\%$ \\
\hline Clinique & $40,0 \%$ \\
\hline Hôpital & $60,0 \%$ \\
\hline Mode d'accouchement : & $\%$ \\
\hline Césarienne & $25,8 \%$ \\
\hline Voie basse & $74,2 \%$ \\
\hline
\end{tabular}

Tableau 3 : répartition selon les Caractéristiques d'accouchement 
- $40 \%$ des femmes enquêtées ont accouché dans des cliniques privées, les autres ont accouché à l'hôpital.

- Près du quart des femmes interrogées ont accouché par césarienne. A ce niveau, l'écart est très important par rapport à la norme internationale. Les publications de l'Organisation Mondiale de la Santé (OMS) expliquent que rien, sur le plan médical, ne saurait justifier de dépasser un taux de naissances par césarienne de 10 à $15 \%$.

\section{Pratique de l'allaitement}

- La mise au sein du nourrisson dans l'heure qui suit la naissance est une pratique propice au bon démarrage de l'allaitement. De même, la mise au sein tardive peut compromettre la poursuite de l'allaitement maternel pour de multiples raisons dont une montée laiteuse insuffisante et des difficultés pour le bébé de prendre le sein. Par conséquent, la proportion des nourrissons mis au sein précocement est un indicateur révélateur de la qualité de la pratique de l'allaitement maternel.

\begin{tabular}{|lr|}
\hline Mise au sein : & $\%$ \\
\hline Le jour de l'accouchement & $49 \%$ \\
\hline 1 jour après l'accouchement & $23 \%$ \\
\hline 2 jours après l'accouchement & $22 \%$ \\
\hline 4 jours après l'accouchement & $19 \%$ \\
\hline Jamais allaité & $7 \%$ \\
\hline
\end{tabular}

Tableau 4 : répartition par délai de la première mise au sein

Il ressort de l'étude que la mise au sein le jour de l'accouchement concerne $34 \%$ uniquement des femmes interrogées alors que plus de la moitié des femmes interrogées n'allaitent que le lendemain de l'accouchement. La mise au sein le jour de l'accouchement aurait connu un recul très significatif par rapport aux résultats de l'enquête nationale sur la population et santé de la famille(ENSFP) réalisée par le Ministère de la Santé (2004) où $89 \%$ des femmes allaitaient leurs nourrissions le jour de l'accouchement.

De même cette tendance baissière s'était déjà confirmée car les résultats de l'ENSFP réalisée par le Ministère de la Santé (2011) révèlent une baisse de 17 points par rapport à ceux de 2004 pour s'établir à 72,2\% des femmes ayant allaité leurs nourrissions le jour de l'accouchement.

$\mathrm{Au}$ vu des bienfaits de la mise au sein précoce qui peut représenter un facteur déterminant pour le choix de l'allaitement exclusif, cette pratique doit être rétablie. A cet effet, il sera opportun d'une part, de sensibiliser les femmes enceintes sur l'importance d'allaiter leur nourrisson le plus tôt possible et d'autre part, de sensibiliser le personnel médical pour promouvoir cette pratique et intervenir de manière effective pour inciter les mères à s'adonner massivement à cette pratique. 


\begin{tabular}{|lr|}
\hline $\begin{array}{l}\text { Motivations pour le choix de } \\
\text { l'allaitement maternel exclusif }\end{array}$ & $\%$ \\
\hline $\begin{array}{l}\text { Bienfaits du lait maternel pour } \\
\text { l'enfant }\end{array}$ & $93 \%$ \\
\hline Relation privilégiée mère enfant & $41 \%$ \\
\hline $\begin{array}{l}\text { Bienfaits de l'allaitement } \\
\text { maternel pour la mère }\end{array}$ & $34 \%$ \\
\hline $\begin{array}{l}\text { Facilité d'utilisation (pas de } \\
\text { stérilisation, ...) }\end{array}$ & $7 \%$ \\
\hline Choix du mari & $3 \%$ \\
\hline Prévention contre les allergies & $3 \%$ \\
\hline $\begin{array}{l}\text { Motivations pour le choix de } \\
\text { l'allaitement mixte }\end{array}$ & $\%$ \\
\hline $\begin{array}{l}\text { Bienfaits du lait maternel pour } \\
\text { l'enfant }\end{array}$ & $75 \%$ \\
\hline Relation privilégiée mère enfant & $74 \%$ \\
\hline $\begin{array}{l}\text { Bienfaits de l'allaitement } \\
\text { maternel pour la mère }\end{array}$ & $27 \%$ \\
\hline $\begin{array}{l}\text { Prévention des coliques du } \\
\text { nourrisson }\end{array}$ & \\
\hline Facilité d'utilisation & $18 \%$ \\
\hline Prévention contre les allergies & $18 \%$ \\
\hline Raisons Economiques & $13 \%$ \\
\hline $\begin{array}{l}\text { Motivations pour le choix de } \\
\text { l'allaitement artificiel }\end{array}$ & $3 \%$ \\
\hline Nourrisson refuse de téter & $23 \%$ \\
\hline Maladie de l'enfant & $22 \%$ \\
\hline Insuffisance du lait maternel & $19 \%$ \\
\hline Problèmes de seins & $7 \%$ \\
\hline Facilité à reprendre le travail & \\
\hline Tableau 5 : motivations par mode & \\
\hline
\end{tabular}

Tableau 5 : motivations par mode d'allaitement

- S'agissant des motivations relatives au choix de l'allaitement maternel exclusif, la grande majorité des femmes enquêtées (93\%) ont opté pour ce mode en raison des bienfaits du lait maternel pour l'enfant. La relation privilégiée mère-enfant (41\%) et les bienfaits de

- l'allaitement maternel pour la mère (34\%) sont également cités parmi les motivations. D'autres motivations beaucoup moins fréquentes sont évoquées à savoir l'influence du mari, la facilité d'utilisation et la prévention contre les allergies. On peut présager que ces femmes sont sensibilisées sur les bienfaits de l'allaitement maternel exclusif pour le nourrisson et optent pour ce mode d'allaitement pour l'intérêt de leur progéniture.

A l'instar de notre étude, l'enquête réalisée par F. Bellati-Saadif et al. (1996) révèle qu'en ce qui concerne les motifs invoqués pour justifier le choix du mode alimentaire, la majorité des femmes questionnées ont répondu que le sein est le mode alimentaire du nourrisson $(69,7 \%)$. En revanche, les 
résultats de F. Bellati-Saadif et al. (1996) révèlent que la tradition familiale vient en deuxième position avec $18,6 \%$ alors que cette motivation n'a pas été évoquée par les femmes que nous avons interviewées.

- Pour les femmes adoptant l'allaitement mixte, elles ont choisi d'allaiter au sein pour les bienfaits du lait maternel pour l'enfant (75\%), pour la relation privilégiée mère-enfant $(75 \%)$, et pour les bienfaits de l'allaitement maternel pour la mère $(27 \%)$.

Par ailleurs, elles ont choisi d'introduire en parallèle le lait industriel principalement pour pouvoir reprendre le travail $(50 \%)$ et/ou s'absenter $(12 \%)$. D'autres motivations justifient cette pratique notamment l'insuffisance du lait maternel (44\%).

Selon F. Bellati-Saadif et al. (1996), en cas d'allaitement mixte, l'insuffisance lactée est évoquée par $87,1 \%$ des femmes.

- Le recours à l'allaitement artificiel semble être une option subie et non choisie délibérément. Ainsi, 7 femmes sur 10 n'ont pas allaité car leur nourrisson refusait de téter $(50 \%)$ ou à cause d'une maladie du nourrisson rendant l'allaitement difficile. L'insuffisance lactée est évoquée par $22 \%$ des femmes interrogées et 2 femmes sur 10 ont eu des problèmes au niveau des seins (abcès, crevasses ...) qui les ont empêché d'allaiter.

Selon les résultats de F. Bellati-Saadif et al. (1996), celles qui ont opté pour l'alimentation artificielle, l'insuffisance lactée est invoquée dans $36,1 \%$ des cas, l'agalactie dans $30,5 \%$ et le refus du lait maternel dans $28,2 \%$. Aucune des mères n'a évoqué le côté esthétique.

En concordance avec les résultats F. Bellati-Saadif et al. (1996), l'insuffisance du lait maternel est respectivement évoquée par $36,1 \%$ des femmes interrogées.

Les résultats de l'enquête sont aussi en phase avec l'ENSFP de 2011 (Ministère de la Santé, 2011) qui révèle que l'insuffisance du lait maternel, le refus du sein et le problème au niveau du sein ou du mamelon sont également évoqués.

La majorité des difficultés ayant entrainé le choix de l'allaitement artificiel sont engendrés par une mauvaise connaissance des pratiques de l'allaitement et pouvaient être écartés aisément avec la mise en place de quelques mesures préventives.

A cet effet, et pour y parvenir, les femmes doivent être sensibilisées au cours de la grossesse sur la manière d'allaiter, les positions favorisant l'allaitement et sur l'importance d'effectuer une mise au sein précoce pour éviter le problème lié au refus du nourrisson de téter. Elles doivent également être informées en amont sur la manière d'entretien et d'hygiène des seins pour éviter les abcès, crevasses qui rendent l'allaitement difficile.

- Les résultats obtenus montrent que plus de la moitié des femmes interrogées allaitent leurs nourrissons à la demande (au moins 6 tétées par 
jour) et 30\% d'entre elles allaitent leurs nourrissons toutes les deux à trois heures. Les résultats de notre enquête convergent avec les résultats de l'ENSFP de 2011 (Ministère de la Santé, 2011) qui révèle que 59,3\% des nourrissons de moins de six mois ont reçu au moins 6 tétées. Ces résultats laissent présager que la fréquence de l'allaitement est satisfaisante au Maroc. - L'âge moyen d'introduction d'une alimentation autre que le lait maternel s'élève à 2,38 mois. Il diffère selon le type d'aliment introduit. L'âge moyen d'introduction du lait industriel quant à lui est estimé à 3 mois avec en moyenne 3 biberons par jour. les autres aliments cités qui ont été introduits avant l'âge de six sont l'eau, le jus d'orange, les compotes de fruit, les tisanes, la soupe ...

\section{Facteurs influençant l'allaitement}

L'analyse inter-factorielle de notre enquête fait ressortir les constats suivants :

- L'âge de la mère est un facteur influençant significativement la prévalence de l'allaitement maternel exclusif.

A cet effet, pour les femmes âgées de moins de 35 ans, la prévalence de l'allaitement maternel exclusif varie entre $40 \%$ et $44 \%$.Par contre, pour les femmes âgées de plus de 35 , la prépondérance de ce mode d'allaitement baisse de plus de 10 points ans pour atteindre $31 \%$ seulement. On peut présager que les aptitudes et conditions physiques des femmes sont altérées avec l'âge. Il ne serait donc pas aisé pour les femmes âgées de plus de 35 ans d'opter aisément pour ce mode d'allaitement.

- Le sexe de l'enfant influence le mode d'allaitement. Ainsi, 40\% des nourrissons de sexe masculin sont allaités exclusivement au sein tandis que la prévalence de l'allaitement exclusif est de 34\% seulement chez les nourrissons de sexe féminin.

Ces résultats sont en phase avec les résultats de F. Bellati-Saadif et al. (1996) qui révèlent que les enfants de sexe masculin $(85,3 \%)$ sont plus allaités au sein que les enfants de sexe féminin $(79,4 \%)$.

L'ENSFP de 2011(Ministère de la Santé, 2011) révèle quant à elle que le sexe de l'enfant ne semble pas affecter la décision d'allaiter.

- Selon l'étude, la parité influence significativement le choix du mode d'allaitement. Ainsi la prépondérance de l'allaitement exclusif chez les multipares dépasse largement celle des primipares. Dans ce sens, plus de la moitié des femmes multipares optent pour un allaitement maternel exclusif contre $15 \%$ des primipares. 


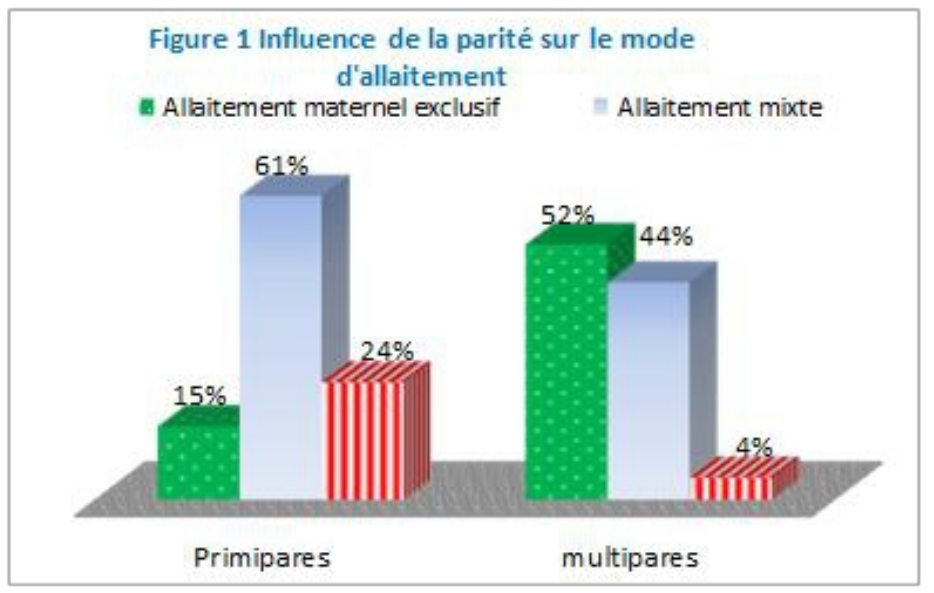

L'allaitement artificiel est pratiqué par $24 \%$ des primipares contre $4 \%$ seulement des multipares.

Ces résultats concordent avec ceux de F. Bellati-Saadif et al. (1996) qui concluent que le rang dans la fratrie influence la pratique de l'allaitement maternel ( $1^{\mathrm{er}}$ rang $76 \%$ et $5^{\mathrm{e}}$ rang $\left.94,6 \%\right)$.

L'étude de Roida et Coll, qui a été réalisée entre le $1^{\text {er }}$ octobre et le 30 novembre 2006, auprès d'un échantillon de 200 couples mère/nouveau-né pris en charge à la maternité Ibn Tofail du CHU de Marrakech au Maroc, révèle également que la fréquence et la durée des tétés sont influencées par la parité. D'ailleurs, 94\% des femmes multipares ont allaité au sein de façon exclusive.
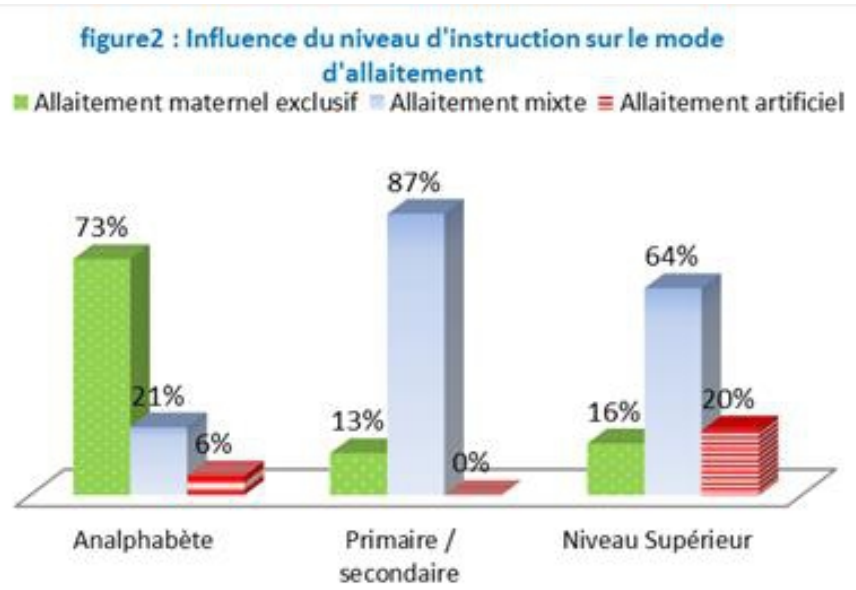

On peut donc présager que la conduite d'une expérience d'allaitement encourage les femmes à opter pour un allaitement maternel, au détriment de l'allaitement artificiel. Dans ce sens, cette différence entre les primipares et les multipares pourrait être expliquée par une habilité et une facilité à allaiter acquises chez les mères multipares. 
- La profession est également un facteur déterminant dans le choix du mode d'allaitement.

L'allaitement maternel exclusif est très prépondérant chez les mères au foyer $(78 \%)$, inexistant chez les femmes exerçant une fonction libérale et très peu adopté par les femmes employés ou fonctionnaires (13\%).

Ces résultats concordant avec ceux de F. Bellati-Saadif et al. (1996) qui révèlent que les femmes au foyer $(84,7 \%)$ allaitent plus que les femmes exerçant un emploi (40\%).

Nos résultats convergent avec ceux de Roida et Coll qui affirment que malgré l'intention de nombreuses femmes de continuer d'allaiter après le retour au travail, l'emploi de la mère constitue un facteur significativement associé à un sevrage précoce.

Les femmes exerçant une fonction libérale justifient ce choix par des contraintes liées à la charge de travail tandis que les femmes employées ou fonctionnaires sont confrontées à des contraintes de temps liées à des horaires de travail inadaptés.

Etant donné que les femmes donnent le biberon à leurs nourrissons par contrainte pour pouvoir reprendre le travail et en vue de la protection, la promotion et le soutien de l'allaitement maternel, il serait opportun que les autorités compétentes prolongent la durée de congé de maternité à six mois au moins puis d'adapter des horaires de travail, pour permettre aux mères actives de prolonger la durée de l'allaitement comme préconisé par l'OMS.

Il est également judicieux que le personnel opérant en milieu médical et hospitalier initie les mères sur les modalités d'extraction et de préservation du lait maternel, à donner au nourrisson en cas d'absence de la mère.

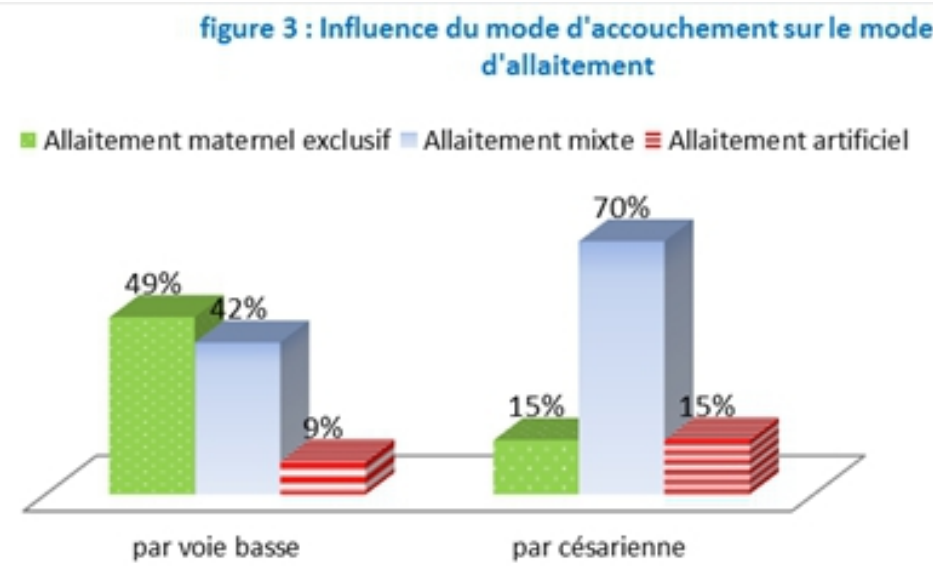

- L'accouchement par voie basse s'avère avoir aussi une influence positive sur le choix d'allaitement maternel exclusif, tandis que l'accouchement par césarienne a été identifié comme un facteur significatif pour le choix de 
l'allaitement mixte. Ainsi, une femme sur deux ayant accouché par voie basse a opté pour l'allaitement maternel exclusif tandis que $15 \%$ seulement des femmes ayant accouché par césarienne ont opté pour ce mode d'accouchement. En revanche, $70 \%$ des femmes ayant subi une césarienne ont opté pour un allaitement maternel mixte. Au regard de ces résultats, une réflexion doit être menée pour baisser le recours à la pratique de la césarienne dans notre pays qui demeure largement plus élevé en comparaison avec les recommandations de l'OMS.

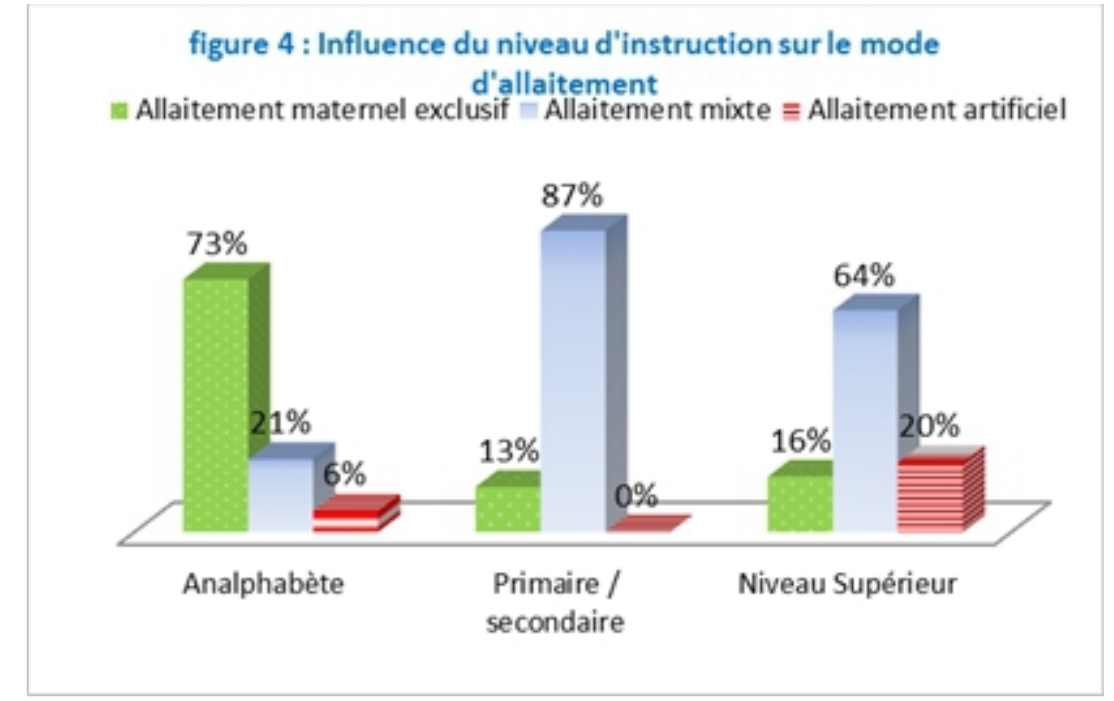

- Les résultats de notre enquête montrent que le mode d'allaitement varierait en fonction du niveau d'études de la mère. L'allaitement maternel exclusif est largement adopté par des femmes analphabètes (73\%) alors que la prépondérance de l'allaitement maternel exclusif ne dépasse pas 16\% chez les femmes ayant un niveau d'étude primaire, secondaire ou supérieur. Néanmoins, selon l'ENSFP de 2011 (Ministère de la Santé, 2011), le niveau d'instruction ne semble pas affecter la décision d'allaiter.

\section{Modalités de sevrage}

- Selon notre étude, la durée moyenne de l'allaitement s'élève à 7,7 mois. Elle diffère selon le mode d'allaitement. Cette durée aurait-elle tendance à se raccourcir? Les enquêtes de l'ENSFP de 2004 (Ministère de la Santé, 2004) et de 2011 (Ministère de la Santé, 2011) révèlent quant à elles respectivement une durée moyenne de l'allaitement de 13,9 de 16,3 mois.

- Le sevrage constitue une étape cruciale dans la vie des nourrissons et doit être menée dans les règles de l'art. Les raisons de sevrage sont diverses, l'insuffisance du lait maternel vient en tête $(53 \%)$ suivi de la facilité de s'absenter ou de reprendre le travail (29\%). D'autres raisons sont évoquées notamment l'atteinte de l'âge de sevrage de deux ans tel que défini par la 
religion, une nouvelle grossesse, la maladie de l'enfant ou de la mère nécessitant la prise d'un traitement, la contrainte de donner plusieurs tétées nocturnes ou quand le nourrisson refuse de téter.

- La même tendance est rapportée par l'ENSFP de 2011 (Ministère de la Santé, 2011). Ainsi, l'insuffisance lactée vient en tête des raisons de sevrage. L'atteinte de l'âge de deux ans par l'enfant, le refus de l'enfant de prendre le sein, la maladie de la mère engendrent également le sevrage.

- Concernant les modalités de sevrage, plus de la moitié des femmes ont arrêté subitement l'allaitement au sein tandis que $36 \%$ des femmes ont opté pour un arrêt progressif de l'allaitement au sein. Dans les autres cas, c'est le nourrisson qui a refusé de téter.

- L'ENSFP de 2011 (Ministère de la Santé, 2011) révèle quant à elle que dans la majorité des cas (84\%), l'interruption de l'allaitement a eu lieu de manière brutale. Les mères adopteraient-elles davantage une interruption progressive de l'allaitement ? Cette amélioration dans les pratiques seraitelle le fruit de plus de sensibilisation des femmes sur la question notamment par les professionnels de la santé ?Au vu de l'incidence physique et psychique de cette pratique sur la santé de l'enfant, le personnel opérant en milieu médical et hospitalier doit s'y attarder sérieusement et accompagner les mères pour que le sevrage se fasse dans les meilleures conditions notamment chez les pédiatres et dans les dispensaires.

\section{Conclusion}

Cette enquête nous a permis d'effectuer plusieurs constats notamment que l'adoption de l'allaitement maternel mixte ou artificiel demeure une option subie plus que choisie et est tributaire de plusieurs facteurs. Ainsi, pour accroitre la prévalence de l'Allaitement maternel, les professionnels de santé peuvent jouer un rôle majeur pour éclairer les femmes sur plusieurs aspects relatifs à cette pratique (mise au sein précoce, positions de l'allaitement, hygiène et entretiens des seins, modalités de sevrage...).

Il est donc judicieux de prendre davantage en compte le rôle des professionnels de la santé à travers des stratégies spécifiques. Il pourrait s'agir notamment de favoriser un contact précoce entre la mère et l'enfant, ou encore de transmettre un discours adapté sur les avantages de l'allaitement maternel afin d'encourager les femmes pour atteindre les recommandations actuelles de santé publique, essentiellement pour l'allaitement exclusif. Par ailleurs, les pères, acteurs-clés de la décision d'allaitement, devraient le plus souvent être associés à ces interventions.

Les variations observées sur l'allaitement selon certaines caractéristiques socioculturelles et démographiques permettent d'identifier des groupes à cibler lors des actions de santé publique visant la promotion de l'allaitement. 
Aussi, malgré les efforts fournis par le Ministère de la Santé et le tissu associatif pour la promotion de l'allaitement maternel exclusif qui se traduisent notamment par l'organisation de la semaine de l'allaitement, on peut constater le manque d'efficience des politiques et plans d'action et le manque d'implication de l'état pour la mise en place de mesures concrètes pour l'incitation des femmes à opter pour l'allaitement maternel exclusif.

Le Ministère de la santé doit donc multiplier les campagnes médiatiques sur les bienfaits $d u$ lait maternel et promouvoir plus particulièrement cette pratique auprès des employeurs publics et privés en montrant l'intérêt économique que représente la pratique d'un allaitement maternel exclusif pour leur entreprise et pour le Maroc de demain.

\section{References:}

1. Barkat A., Lyaghfouri A., Mdaghri Alaoui A., \& Lamdouar Bouazzaoui N. (2004). Une réflexion sur l'allaitement maternel au Maroc, Service de Néonatologie, Centre National de Référence en Néonatologie, Hôpital d'Enfants Rabat, Direction de la Population, Ministère de la Santé - 15 juillet 2004.

2. Bellati-Saadif., Sallm. G., Martins. L., Azondekona., \& Kuakuvi N. (1996). Situation actuelle de l'allaitement maternel dans la région d'Agadir au Maroc à propos d'une enquête chez 220 mères, Médecine d'Afrique Noire : 1996, 43 (4).

3. Duijts L., Ramadhani M-K. \& Moll H-A (2009). Breastfeeding protects against infectious diseases during infancy in industrialized countries. A systematic review. Matern Child Nutr. 2009;5(3):pp199210.

4. Elbakali M. (2011). La pratique de l'allaitement maternel au niveau de 3 maternités : situation actuelle et facteurs influençant enquête auprès de 227 mères et 61 professionnels de santé, Rabat, $168 \mathrm{pp}$.

5. Hassani A., Barkat A., Souilmi F-Z., Lyaghfouri A., Kabiri M., Karboubi L., Alaoui I., Lamdouar-Bouazzaoui N. (2005). La conduite de l'allaitement maternel. Étude prospective de 211 cas à la maternité Souissi de Rabat, Journal de pédiatrie et de puériculture, $2005 ; 18$ : pp 343-348.

6. Lara-Villoslada .F., Olivares M., Sierra S.., Rodríguez JM., Boza J. \& Xaus J. (2007). Beneficial effects of probiotic bacteria isolated from breast milk, British Journal of Nutrition, Volume 98, October, pp 96-100.

7. Kersuzan C., Gojard S., Tichit C., Thierry X., Wagner S., Nicklaus S., Geay B., Charles M-A., Lioret S. \& de Lauzon-Guillain B. (2014). Prévalence de l'allaitement à la maternité selon les 
caractéristiques des parents et les conditions de l'accouchement. Résultats de 1'Enquête Elfe maternité, France métropolitaine, 2011, Bulletin Epidémiologique Hebdomadaire - BEH, Saint-Maurice (Val de Marne) : Institut de veille sanitaire, 2014, pp440-449.

8. Kramer M-S., Aboud F., Mironova E., Vanilovich I., Platt R-W., Matush L., Igumnov S., Fombonne E., Bogdanovich N., Ducruet T., Collet J-P., Chalmers B., Hodnett E., Davidovsky S., Skugarevsky O., Trofimovich O., Kozlova L. \& Shapiro S. (2008). Breastfeeding and child cognitive development: new evidence from a large randomized trial. Arch Gen Psychiatry. 2008 May;65(5):578-84. doi: 10.1001/archpsyc.65.5.578.

9. Labbok M-H (2001). Effects of breastfeeding on the mother. Pediatr Clin North Am. 2001;48(1): pp 143-58.

10. Ministère de la Santé (2004). Enquête nationale sur la population et santé de la famille- 2011. Récupérée de http://www.sante.gov.ma/.

11. Ministère de la santé au Maroc (2005). Enquête sur la population et la santé familiale (EPSF) (2003-2004), Rabat, pp 241.

12. Ministère de la Santé (2011). Enquête nationale sur la population et santé de la famille- 2004. Récupérée de http://www.sante.gov.ma/.

13. Organisation Mondiale de la Santé (2001). Recommandations de l'OMS en matière d'alimentation du nourrisson, 01 mai 2001, http://www.who.int. 